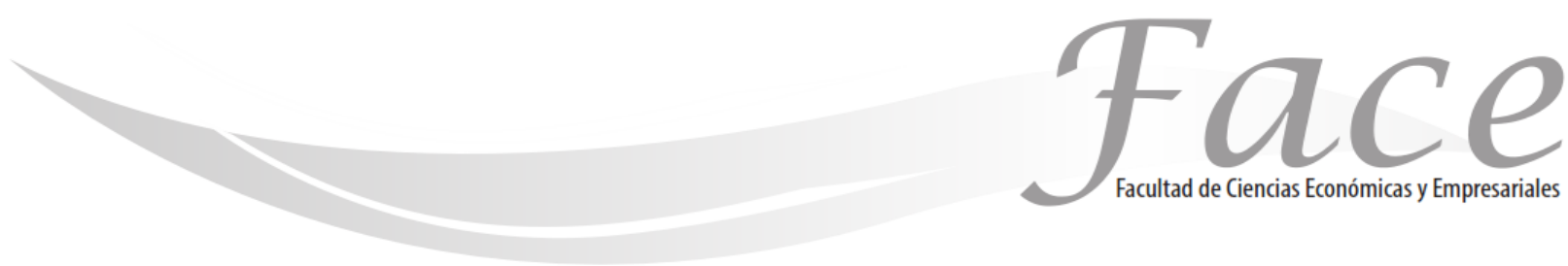

ISSN Impreso: 1794-9920

ISSN Electrónico: 2500-9338

Volumen $17-\mathrm{N}^{\circ} 2$

Año 2017

Págs. $68-80$

\title{
TENDENCIA DE INVESTIGACIÓN CONTABLE EN COLOMBIA: TEORÍA O PRÁCTICA, UNA MIRADA DESDE LAS REVISTAS CONTABLES COLOMBIANAS 2011 A 2015 *
}

\author{
Jenny Paola Beltrán Beltrán ** \\ Enlace ORCID: http://orcid.org/0000-0001-7217-2846 \\ Luis Alfonso González Feliciano *** \\ Enlace ORCID: http://orcid.org/0000-0003-0470-0752
}

Fecha de Recepción: noviembre 92016

Fecha de Aprobación: febreo 142017

\section{Resumen:}

El presente documento pretende identificar la tendencia de investigación en Colombia; teoría y práctica, a partir de la revisión entre el 2011 a 2015 de las revistas indexadas, relacionando temas de las ciencias económicas, administrativas y contables, los cuales abordan temas relacionados a las líneas de investigación en contabilidad. En este sentido, lo que se pretende demostrar a partir del desarrollo del documento es hacia cual tendencia está más inclinada la investigación contable en Colombia; sí está encaminada a desarrollo de fundamentos teóricos de la ciencia contable o sí en definitiva se relaciona a temas de carácter aplicado o práctico, dando solución desde la contabilidad a problemáticas de carácter social y empresarial.

Palabras Claves Investigación contable, Revistas indexadas, teoría contable, práctica contable.

*Contador Público de la Universidad Militar Nueva Granada, Estudiante de Maestría en Administración Financiera de la Universidad Sergio Arboleda, Bogotá. Asistente de Investigación e Integrante del grupo de investigación INCONDIS del programa de Contaduría Pública de la Facultad de Estudios a Distancia de la Universidad Militar Nueva Granada, Bogotá Colombia. Correo electrónico: tmp.jenny.beltran@unimilitar.edu.co

${ }^{*}$ Contador Público. Magister en Educación. Especializado en Docencia Universitaria y Control Interno. Docente tiempo completo e integrante del grupo de investigación INCONDIS del programa de Contaduría Pública de la Facultad de Estudios a Distancia de la Universidad Militar Nueva Granada Correo electrónico: Luis.gonzalez@unimilitar.edu.co 


\begin{abstract}
This document aims to identify the research trend in Colombia; Theory and practice, from the review of indexed journals between 2011 and 2015, relating issues of economic, administrative and accounting sciences, which address issues related to research lines in accounting. In this sense, what is intended to demonstrate from the development of the document is towards which trend is more inclined accounting research in Colombia; It's aimed at developing theoretical foundations of accounting science or if it's related to issues of an applied or practical, giving a solution from accounting to social and business issues.
\end{abstract}

Keywords: Accounting research, Journals indexed, accounting theory, accounting practice.

\title{
TENDENCIA DE INVESTIGACIÓN CONTABLE NA COLÔMBIA: TEORÍA O PRÁCTICA, UNA MIRADA DESDE LAS REVISTAS CONTABLES COLOMBIANAS 2011 A 2015.
}

\section{Resumo}

Este documento tem como objetivo identificar a tendência de pesquisas na Colômbia; teoria e prática, com base na revisão 2011-2015 de revistas indexadas, relacionando questões de ciências econômicas, administrativas e contábeis, que tratam de questões relacionadas com a pesquisa em contabilidade. Neste sentido, o que é demonstrar a partir do desenvolvimento do documento é em direção a essa tendência é mais inclinado contabilidade pesquisa na Colômbia; si visa o desenvolvimento dos fundamentos teóricos da ciência ou contabilidade própria, em última análise se refere a questões de aplicada ou prática, fornecendo soluções de contabilidade para questões de natureza social e de negócios.

Palavras-chave contabilidade Research, arbitrou revistas, representando teoria, representando prática. 


\section{INTRODUCCIÓN:}

En las últimas décadas en Colombia se ha venido generalizando la preocupación y la discusión sobre la investigación contable, pues esta, se ha en caminado en varias perspectivas que se sustentan en el desarrollo teórico y técnico de la disciplina y la profesión. En este sentido, la investigación contable se ha perfilado como una estrategia pedagógica importante en la formación de contadores públicos para que trasciendan las capacidades técnico-instrumentales y puedan enfrentar las demandas del contexto social y organizacional (Muñoz y otros, 2015).

Si se parte de la premisa acerca de la investigación como una práctica científica dirigida a ampliar las fronteras del conocimiento, para franco (1998) la investigación en contabilidad en Colombia conduce a trabajar un concepto más laxo definiéndolo como la búsqueda de respuestas a interrogantes planteados a nivel científico, técnico o simplemente monográfico. Para Gonzales (2005) la investigación contable debe ser asumida como tarea permanente que impulsa de manera constante el desarrollo de las disciplinas, las profesiones y por consiguiente la investigación como actividad humana no debe reducirse a la vida académica, es decir, la investigación debe permanecer durante toda la vida profesional. En este sentido, la investigación contable no solamente debe estar encaminada a la reflexión, también debe ir acompañada de instrumentos metodológicos integrados con procesos de análisis para darle solución a problemáticas que surgen de la realidad.

Por su parte, Salazar (2015) argumenta que dentro del desarrollo histórico de la contabilidad se repercute en el desarrollo de la investigación contable, los cuales radican principalmente en cuatro aspectos fundamentales: La falta de leyes o un marco teórico formal, la mirada de un nuevo universo contable por partes (macro, micro contabilidad), separación de los contadores en prácticos y académicos, problemas de medición y valoración, en otras palabras, en la mecanización del saber contable.

El presente documento se desarrolla de la siguiente manera; en primer lugar se abarcan los enfoques de investigación en contabilidad y el desarrollo histórico de las revistas de investigación en la ciencia contable en Colombia, en segundo lugar se habla acerca de la metodología utilizada, la descripción de la muestra seleccionada. En tercer lugar, se muestra los resultados obtenidos y se describe la tendencia de investigación que ha tendido Colombia durante 2011-2015, a partir de las variables utilizadas. En cuarto lugar, se muestran los resultados obtenidos y la discusión. Por último, se exponen las respectivas conclusiones del documento.

\section{MARCO TEÓRICO:}

\section{ANTECENTES DE INVESTIGACIÓN EN LA CONTABILIDAD.}

Naranjo (2015) argumenta que la investigación contable en el contexto de la globalización, ha desarrollado tendencias que pretenden responder a las exigencias que surgen por lo cambios sociales adoptados a nivel económico, político y cultural dentro de los marcos nacional e internacional. Además, la investigación en contabilidad también ha generado debates relacionados acerca de: sí la contabilidad puede considerase una disciplina o ciencia social, a partir de las distintas definiciones, funciones, métodos (el cual permite identificar la postura que toma la investigación) y desde el mismo papel que juega la contabilidad en la sociedad. En la presente sección se tratará de describir algunas tendencias a nivel internacional que se han destacado en la construcción de la investigación en contabilidad.

\subsection{Mirada Ortodoxa y Heterodoxa.}

Mardones (1991, citado por Machado, 2012), afirma que la investigación en contabilidad actualmente se debate entre dos miradas, por un lado, se encuentra la mirada ortodoxa, (principal, positivista, tradicionalista, empírica o funcional) y por el otro lado se encuentra la mirada heterodoxa (alternativa).

La mirada ortodoxa contiene cuatro aspectos característicos; la auténtica explicación científica, el modelo o canon de las ciencias naturales exactas, la explicación relación causa-efecto y el efecto en los fenómenos con el fin de controlar y dominar la naturaleza.

Además esta mirada también denominada "investigación aplicada", se preocupa por las necesidades y funcionales del entorno en el que opera la contabilidad, es decir, con las necesidades de información, control, medición y representación de la riqueza en organizaciones específicas como la empresa, el sector gubernamental y la nación (Gómez, 2003). Por esa misma línea Romero (2014, citado por Gómez, 2003) afirma: "el lado aplicado la investigación se torna pragmática, instrumental y tecnológica, buscando soluciones concretas para resolver problemas de información concretos derivados de investigaciones empíricas, vía inductiva, partiendo del estado de cosas que existen, sin pretender transformarlo"( $\mathrm{p} 10)$.

Por otro lado, la mirada heterodoxa que filosóficamente proviene de la teoría social moderna en las figuras de Karl Marx y Wilhem Dilthey, se denominan alternativas porque: "Quienes adhieren a esta línea de pensamiento, proponen 
Jenny Paola Beltrán Beltrán -Luis Alfonso González Feliciano-

un análisis crítico y socio-histórico de la contabilidad, en el cual se tendrán en cuenta tanto las condiciones en las que se lleva adelante la práctica contable, como las consecuencias de la misma. (Aquel \& Cicerchia, 2012, p 2) Además, esta mirada comprende que la investigación contable se ha clasificado en: interpretativa, critica y las relaciones del poder con la contabilidad (Aquel \& Cicerchia, 2012) y (Larrinaga, 1999). La investigación interpretativa no está interesada en la resolución de problemas, trata de comprender los fenómenos de interacción entre personas en situaciones sociales y su fundamento filosófico basándose en el comportamiento humano y es completamente ajeno a los fenómenos físicos (Larrinaga, 1999). La investigación critica se interesa por el cambio, los conflictos, la coacción y tiene raíces marxistas; presupone que en el mundo existe una desigualdad estructural básica que se reproduce a través de las generaciones y que genera un conflicto entre la clase favorecida y oprimida (Larrinaga, 1999).

\subsection{Los programas de investigación}

Para Cañibano y Angulo (1996) una mirada funcional de la investigación contable se puede clasificar en diferentes programas: legalista, económico y formalizado. El programa legalista nace de la necesidad de regular la contabilidad a través de códigos de comercio, leyes y principios generalmente aceptados 0 de estándares internacionales. Pero Montes et.al (2005) argumenta que la pertinencia de la implementación de la normatividad internacional en la contabilidad colombiana, debe ser basada en la realidad económica del país. Además, evalúa las implicaciones de este hecho como: En primer lugar, la pérdida de la autonomía para emitir normas contables. En segundo lugar, la estandarización contable no se aplica de manera universal porque su economía es dependiente y no tiene punto de comparación con la de los países desarrollados.

Para el programa económico nace de la necesidad de obtener información de la organización tal y como es la teoría positiva de la contabilidad. El programa formalizado nace con la necesidad de los investigadores de aprovechar las ventajas que proporciona una metodología hipotéticodeductiva, es decir su énfasis es la materialización del conocimiento contable con el fin de encontrar la aceptación del saber científico. En el grafico 1, se describen los programas de investigación contable con más detalle.

llustración 1. Programas de investigación contable.

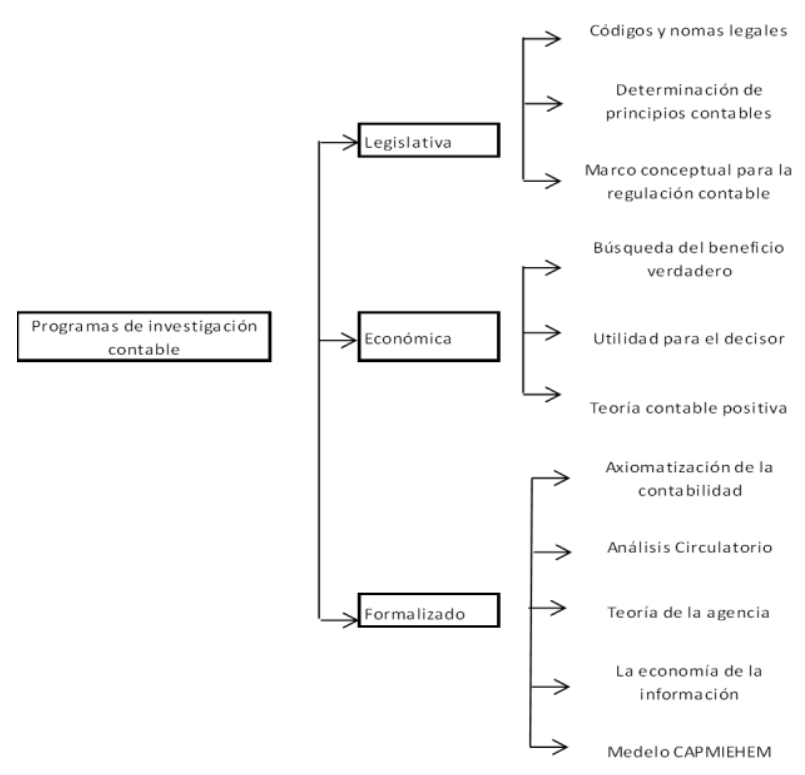

Fuente: Elaboración propia a partir de Cañibano \& Angulo (1996).

\subsection{La contabilidad como ciencia social.}

Larrinaga (1999) toma una postura en donde la contabilidad es vista como ciencia social, es decir, garantiza el funcionamiento de la empresa, genera conocimientos conexos entre la economía y las finanzas, cuantifica el patrimonio público o privado, determina las pérdidas y ganancias de las personas naturales y jurídicas y precisa las responsabilidades de los contribuyentes, así mismo, sistematiza la política tributaria relacionada con la estructura formal y funcional del sistema impositivo, su distribución social, los efectos de los tributos y su utilización como instrumentos dinámicos para comprender el subsistema económico y su incidencia en el desarrollo nacional.

La contabilidad al tomar la postura de ciencia social, se fundamenta en vectores descritos por Millan (1999), estos son; el sujeto y objeto, conceptos teóricos, métodos y teoría. Dichos vectores se desarrollan a continuación:

De acuerdo con Larrinaga (1999), el objeto es visto en lo social como una realidad concreta y empírica que es independiente, por esto su conocimiento puede adquirirse mediante la observación, en donde las experiencias de los individuos están completamente restringidas por el entorno social. Por otra parte, lo subjetivo, entendido como la realidad social que existe únicamente como un proceso de

entendimiento individual que se ha creado a partir de conceptos y etiquetas que le ayudan a interpretar la realidad, establece que el conocimiento científico solo se 
puede adquirir de forma personal y es suficientemente autónomo y libre de recrear su propio entorno.

Los conceptos teóricos son las unidades de la estructura lógica del pensamiento. Además se consideran como:

"Los modos en que el científico ha aprendido a comprender los fenómenos complejos, a darse cuenta de sus relaciones mutuas y a representarlos en forma comunicable. Entre las cosas más maravillosas que consideramos inventadas por la ciencia se encuentran sus conceptos, pues constituyen, de hecho, la elaborada instrumentación y la alta tecnología del pensamiento y del discurso científico" (Wortojsky, 2000, citado por Torres \& Lam, 2012).

Por ejemplo los conceptos como partida doble, debe, haber, débito, crédito, auditoría, tributación, costo, presupuesto, estados financieros, impuesto, son entre otros, conceptos particulares de la ciencia contable. La Contabilidad establece su campo de acción científico, que comprende: balance económico general de la empresa, información financiera, y manejo de la economía de la empresa (Torres \& Lam, 2012).

Para Córdoba (2010), los métodos existen a partir de una discusión acerca de la discusión del método de las ciencias naturales por parte de las ciencias sociales; se identifican métodos positivos y métodos normativos. Así mismo, se mencionan herramientas metodológicas sincrónicas (deducción e inducción) y diacrónicas (Programas de investigación con base en Lakatos, paradigmas con base Kunh, redes teóricas con base en Stegmuller y Sneed) con las cuales se evalúa el proceso científico con la disciplina contable. Sin embargo, para Moya (2005) en el área de las ciencias sociales existen diferentes métodos de investigación alternativos para llevar a cabo una investigación empírica. De esta manera, es posible el uso conjunto de más de un método de investigación, combinando metodología cuantitativa y cualitativa para incrementar la fiabilidad de los estudios realizados. La elección de uno u otro método dependerá, entre otras variables, de las características y la naturaleza de la investigación.

Por último, se destaca la teoría, la cual "es el conjunto de hipótesis sucesivas demostradas y comprobadas por uno 0 varios investigadores que estudian el mismo "problema objeto de estudio" con los mismos o diferentes métodos, técnicas, estrategias e instrumentos de investigación; elevando los conocimientos adquiridos durante el proceso de investigación a un nivel de abstracción científica. La teoría contable plasma el conocimiento teórico-práctico

para obtener rentabilidad y desarrollo empresarial". (Torres \& Lam, 2012). Sin embargo varios autores han detectado la ausencia de teorización en el campo contable y la consideran obstáculo para que la contabilidad logre el estatus de ciencia, sin desconocer los avances teóricos tanto a nivel nacional como internacional con elementos positivos, normativos y positivo-normativos (Córdoba, 2010).

1.4 Perspectivas alternativas de investigación en Contabilidad (Larrinaga, 1999)

Desde la postura de Larrinaga (1999), la investigación contable promueve un importante aporte al fortalecimiento del pensamiento contable realizando una caracterización de los trabajos investigativos y los clasifica en: Estudios interpretativos, prospectiva crítica, relación entre contabilidad-poder y perspectiva dominante, identificando en cada una de las clasificaciones, los supuestos epistemológicos, ontológicos, metodológicos, creencias sobre la realidad física y social, y la relación entre teoría y práctica, partiendo de trabajos de Chua (1986), Laughlin y Lowe (1990) y Puxty (1993).

Este trabajo corresponde a una ruta que permite comprender los desarrollos en la contabilidad desde las diferentes perspectivas que se describen a continuación:

\subsubsection{Los Estudios interpretativos:}

Se caracterizan porque no están interesados en la resolución de problemas, su objeto de conocer no es actuar, como podría ocurrir en la teoría de la contingencia, donde se estudia la relación idónea entre entornos y sistemas de información. El investigador no plantea los problemas sino que trata de estudiar su naturaleza como un fenómeno más de la realidad social. También, la investigación interpretativa trata de comprender los fenómenos de interacción entre personas en situaciones sociales y su fundamento filosófico en el comportamiento humano que es completamente ajeno a los fenómenos físicos.

Puxty (1993, citado por Larrinaga, 1999, p.114) argumenta que los estudios interpretativos deben estar encaminados a:

1. "Los seres humanos actúan sobre las cosasobjetos, gente, organizaciones, ideas abstractas, actividades 0 situaciones en función de los significados que tiene para ellos"

2. "Los significados que las cosas tiene para los individuos son los resultados de la interacción social, es decir, las cosas no tienen un significado objeto anterior, independiente y extremo al sujeto"

3. "Esos significados son manejados y modificados a través de un proceso interpretativo que se utiliza por cada individuo para tratar los signos que encuentra. Los significados no están ahí, sino que el individuo permanece activo en un proceso de interpretación." 
De acuerdo con Larrinaga (1999), las ideas antes expuestas tienen consecuencias sobre el estudio de la contabilidad, pues el trasfondo que tienen las prácticas contables, es resultado de la interacción social, es decir, que la información puede tener diferentes significados en función de los contextos sociales, políticos e históricos. Por lo tanto las prácticas contables forman parte de una representación social, que está siendo continuamente recreada, esto abre campo a la teoría de las representaciones sociales, la cual es considerada como uno de los enfoques más importantes para representar las formas del conocimiento cotidiano. Así mismo, el conocimiento elaborado en la interacción de las prácticas sociales, en definitiva constituye sistemas cognitivos en los que es posible conocer estereotipos, opiniones, creencias valores y normas, aunque puede decirse que las representaciones sociales son un concepto problemático y complejo, dado que los principales objetos y sujetos de su análisis son los seres humanos (Amaya, 2002).

Las representaciones sociales se construyen a partir de una serie de materiales de diversas procedencias:

- $\quad$ En el conjunto de prácticas sociales que se encuentran relacionadas con las diversas modalidades de la comunicación social. En efecto los procesos de comunicación social, donde se origina principalmente la construcción de las representaciones sociales. En ese sentido, los medios de comunicación de masas tienen un peso preponderante para trasmitir valores, conocimientos creencias y modelos de conductas.

- $\quad$ El fondo cultural acumulado en la sociedad a lo largo de su historia: Dicho fondo está constituido por las creencias ampliamente compartidas, los valores considerados como básicos y las referencias histórica y culturales, que conforman la memoria colectiva y la identidad de la propia sociedad. Todo ello se materializa en las divisas instituciones sociales, de acuerdo con lbañes (citado por Amaya, 2002), las fuentes de determinación de las representaciones sociales se encuentran en el conjunto de condiciones económicas, sociales e históricas que caracteizan a una sociedad determinada y el sistema de creencias y de valores que circulan en su entorno.

\subsubsection{Perspectiva critica}

La perspectiva crítica se interesa por el cambio, los conflictos, la coacción y tiene raíces marxistas. Presupone que en el mundo existe una desigualdad estructural básica que se reproduce a través de las generaciones y que genera un conflicto entre la clase favorecida y la clase oprimida. Por su parte, Karl Marx y Wilhem Dilthey, son considerados como los grandes exponentes. Sus ideas dan frutos en el nacimiento de la sociología de corte socialista en el caso de Marx, mientras que la influencia de Dilthey es notable en la corriente sociológica alemana de fines de siglo XIX siendo Weber el autor para quien la comprensión racional y evidente será la condición ontológica de la vida humana en sociedad. (Arquel y cicerchia 2012).

Peter Miller (1994, citado por Arquel \& cicerchia 2012), afirma que en 1981 se produce una transformación en la manera de entender a la contabilidad; desde entonces y en adelante las prácticas contables dejan de ser consideradas principalmente en sus aspectos organizacionales, esto es gracias a un congreso llevado a cabo en la Universidad de California en la ciudad de Los Ángeles en Julio de 1981, en donde Anthony Hopwood presenta en su trabajo titulado "el rol que tiene la contabilidad en la organización y la sociedad", en el cual concluye que la contabilidad nunca puede ser vista en términos puramente organizacionales. A partir de ese momento, Anthony Hopwood es considerado como uno de los grandes exponentes de dicha corriente en el campo de la contabilidad.

Por otro lado, los aportes de Mattessich (2006, citado por Arquel y cicerchia 2012), también son considerados para exponer la corriente crítica interpretativa, Mattessich considera que dicha corriente es fuertemente influenciada por la sociología, la psicología y la filosofía, y le asigna un rol de crítica a la economía neoclásica y liberal, inclinando sus preferencias políticas hacia el socialismo. Mattessich (2006) hace especial énfasis en la contabilidad vista como tecnología, asignando un nuevo rol a la contabilidad, dando como resultado una materia vital para todas las esferas de la vida social, ya que se constituye en una práctica intrínseca y constitutiva de las relaciones sociales.

\subsubsection{Relación entre poder y contabilidad}

Foucautl (1991, citado por Ortega, 2013) considera que la contabilidad es un dispositivo de poder que responde a las necesidades y a los fines del poder capitalista, es decir, la contabilidad puede servir como instrumento estratégico para la organización de los medios humanos o de la fuerza de trabajo desde el punto de vista de su eficiencia, en otras palabras sirve como instrumento para vigilar, controlar e imponer disciplina a la fuerza de trabajo. A partir de estas consideraciones se crean dos categorías para el análisis de la contabilidad como dispositivo del poder, por un lado está el poder disciplinario y por el otro la contabilidad en el gobierno directo y distributivo.

El poder disciplinario puede evidenciarse desde los diversos casos que expone Macintosh (2002, citado por Ortega, 2013), que se refieren, principalmente, al poder que ejerce la contabilidad por medio de prácticas modernas como el presupuesto y el costeo estándar, y de informes como los reportes financieros, el estado de ganancias y pérdidas y los planes expresados en números contables. 
De acuerdo al planteamiento de Chua \& Neu (1997, citado por Ortega, 2013), la contabilidad participa de dos formas en el gobierno indirecto de la vida de los hombres y el gobierno distributivo. En el gobierno indirecto se da mediante el cálculo de los costos para determinar cómo y qué servicios producir, la otra se presenta mediante la alineación (bajos costos) de la conducta económica, social y personal de ciertos individuos y/o instituciones con los objetivos políticos y sociales. Para el gobierno distributivo, es la selección de las tecnologías que le permitan al Estado distribuir entre cada uno de los individuos de la población las cosas que han sido dispuestas, es decir el Estado determina las cosas para disponer y a quién distribuírselas 0 asignárselas.

En el siguiente capítulo se presenta el desarrollo que han tenido las revistas de investigación contable en Colombia, se exponen algunos debates relacionados al impacto de las publicaciones en investigación contable, por último, se evidencian algunas políticas exigidas por COLCIENCIAS para las publicaciones científicas.

\section{METODOLOGÍA:}

La investigación propuesta puede clasificarse como descriptiva, en cuanto a sus objetivos y revisión documental, en cuanto a los procedimientos. Se propone el análisis de revistas para la posterior elaboración de las respectivas estadísticas, lo cual reafirma el carácter descriptivo de la investigación, que busca describir, clasificar e interpretar los datos recolectados acerca de la producción en investigación contable en Colombia.

\subsection{La muestra}

Las nuevas exigencias por parte de COLCIENCIAS, en cuanto a la indexación de las revistas para el año 2016 son mucho más estrictas, pues se crearon políticas para mejorar la calidad de las publicaciones científicas nacionales y estas sean visibles en ISI-Scopus, el primer objetivo al que le apuesta COLCIENCIAS (2016) es de aumentar la participación de los investigadores nacionales en revistas JCR y SJR, la segunda apuesta es aumentar la participación de las revistas científicas nacionales en estas mismas bases de datos. Con la implantación de este modelo Macías (2016) argumenta que:

"El nuevo modelo de clasificación propuesto implica cambios radicales, ya que las revistas serían $A 1$ solo si están en los cuartiles Q1 y Q2 de JCR o SJR y para llegar a A2 habría que estar en los cuartiles Q3 y Q4 de JCR o SJR; la clasificación en B y C dependerá de cuartiles construidos para cada gran área del conocimiento, con base en las citas registradas en Google Académico" (p.41). A partir de este modelo encontró que para 2016 en Colombia solo hay dos revistas dedicadas a la publicación de la disciplina contable, las cuales son: Cuadernos de contabilidad de la Pontificia Universidad Javeriana con categoría A2 y LÚMINA de la Universidad de Manizales categorizada en C. Además, se encontró que hay 20 revistas indexas relacionados a temas relacionados a las ciencias economías, sin embargo, para la muestra se seleccionó las revistas que se presentan la tabla 1. Dentro de la muestra seleccionada de las revistas y entre el periodo seleccionado, se encontró que hay 265 artículos los cuales son la base de estudio del presente documento.

\section{Tabla 1. Listado de revistas seleccionadas para la investigación.}

\begin{tabular}{|c|c|c|c|}
\hline $\begin{array}{l}\text { Categoria } \\
2016 \\
\end{array}$ & $\mid \begin{array}{c}N \\
\text { Indexación }\end{array}$ & Nomble & Univer siclaal \\
\hline A1 & 0121.5051 & Revista Innovar & $\begin{array}{l}\text { Universidad Nacional de } \\
\text { Colombia }\end{array}$ \\
\hline A2 2 & $0121-6805$ & Investigación y reflexión & Universidad Militar \\
\hline A2 & 0123-1472 & Cuadernos de Contabilidad & Pontificia Universidad \\
\hline c & 17949920 & $\begin{array}{l}\text { Revista de la facultad de Ciencias } \\
\text { Económicasy Empresariales de la } \\
\text { Universidad de Pamplona }\end{array}$ & Universidad de Parrplona \\
\hline c & $1794-7561$ & $\begin{array}{l}\text { Revista de economia y } \\
\text { administratión }\end{array}$ & $\begin{array}{l}\text { Universidad Autónoma de } \\
\text { Otcidente }\end{array}$ \\
\hline c & $2145-6194$ & $\begin{array}{l}\text { Revista civilizar de Empresa y } \\
\text { Economia }\end{array}$ & Universida Sergio Arboleda \\
\hline c & $2215 \cdot 8405$ & TEUKEN BIOIKAY & $\begin{array}{l}\text { Politécrico Colombiano } \\
\text { Jaime Isaza Cadavid }\end{array}$ \\
\hline $\begin{array}{l}\text { c } \\
\text { c }\end{array}$ & $\begin{array}{l}2145-5147 \\
0123-4072\end{array}$ & $\begin{array}{l}\text { Desartollo Gerential } \\
\text { Revisla LUMINA }\end{array}$ & $\begin{array}{l}\text { Universidad Simón Bolivar } \\
\text { Universidad De Manizales }\end{array}$ \\
\hline c & $2346 \cdot 3279$ & EN CONTEXTO & $\begin{array}{l}\text { Tecnológico de Antioquia } \\
\text { Institución Universit aria }\end{array}$ \\
\hline
\end{tabular}

Fuente: Elaboración propia

\subsection{Análisis de la información}

Para la construcción de la matriz de análisis de datos se tuvieron en cuenta variables como, idioma en la que se publicó el artículo, universidad o entidad quien financio la investigación, líneas de investigación y la selección de las líneas se realizó con base a las líneas utilizadas por los grupos de investigación contable Colombianos, estos son: Auditoria, contabilidad ambiental y social, contabilidad de gestión, contabilidad financiera, contabilidad internacional, contabilidad pública, Investigación y educación, teoría contable, tributación (Valero y Patiño, 2012, citado por Macías y Patiño, 2014). Dentro de la variable "revistas" se tiene en cuenta el número de publicaciones en las líneas descritas con anterioridad en cada una de las revistas, por último se destaca la variable "Publicaciones" en donde se evidencian el número de publicaciones totales realizada entre 2011 a 2015.

El rigor del presente documento se presenta en la variable a cerca de las "metodologías de investigación utilizadas" y se tomaron a partir del libro metodología de investigación de Sampieri (1996) las cuales son: los estudios de caso, documento de revisión, documento de reflexión, estudio cuantitativo. Cabe aclarar que la categoría de metodología de investigación se construye a partir de las metodologías utilizadas en cada artículo revisado y se describen a continuación: 


\section{Estudios de caso}

La metodología de estudio de caso es una herramienta valiosa de investigación, y su mayor fortaleza radica en que a través del mismo se mide y registra la conducta de las personas involucradas en el fenómeno estudiado, mientras que los métodos cuantitativos sólo se centran en información verbal obtenida a través de encuestas por cuestionarios (Yin, 1989, citado por Martínez, 2011). Además, en el método de estudio de caso, los datos pueden ser obtenidos desde una variedad de fuentes, tanto cualitativas como cuantitativas; esto corresponde a, documentos, registros de archivos, entrevistas directas, observación directa, observación de los participantes e instalaciones u objetos físicos (Chetty, 1996).

Yin (1989, citado por Martínez, 2011) considera el método de estudio de caso apropiado para temas que se consideran prácticamente nuevos, pues en su opinión, la investigación empírica tiene los siguientes rasgos distintivos; examina 0 indaga sobre un fenómeno contemporáneo en su entorno real, las fronteras entre el fenómeno y su contexto no son claramente evidentes, se utilizan múltiples fuentes de datos, y puede estudiarse tanto un caso único como múltiples casos.

\section{Documentos de revisión}

Este tipo de documentos tiene como propósito presentar una síntesis de las lecturas realizadas durante una base de investigación documental, la metodología propuesta para la revisión bibliográfica puede ser aplicada a cualquier tema de investigación para determinar la relevancia e importancia del mismo y asegurar la originalidad de una investigación. Además, permite que otros investigadores consulten las fuentes bibliográficas citadas. (Luna et.al, 2014), esto se realiza con el fin de dar fundamento teórico a la investigación realizada.

\section{Documentos de reflexión}

Esta metodología presenta resultados de una investigación determinada desde una perspectiva analítica, interpretativa o crítica del autor, sobre un tema específico, recurriendo a fuentes originales. Este tipo de documento se conoce en el ámbito académico como ensayo. En general son escritos que mediante una estructura identificable exponen con claridad un tema, desarrollan unas implicaciones y presentan unas conclusiones.

\section{Estudio cuantitativo}

El estudio cuantitativo se caracteriza por ser un tipo de artículo de investigación científica, el cual involucra una metodología, resultados, discusión y conclusiones, es decir, el artículo presenta de forma detallada los resultados originales del proyecto. De acuerdo a Martínez (2011), las investigaciones cuantitativas se basan en un número elevado de observaciones, las cuales pueden ser

descriptivas y explicativas, por cuanto se muestran adecuadas para el uso de técnicas estadísticas de carácter descriptivo, tales como: la tabla de frecuencia y las medidas de tendencia central, a través de las cuales se explica en ejemplos como: el análisis de regresión y el análisis de varianza entre otras técnicas, que permiten determinar los factores que ejercen influencia significativa en el fenómeno objeto de estudio.

\section{RESULTADOS Y DISCUSION:}

La investigación contable en Colombia se ha venido consolidando en los últimos años, gracias a reflexiones desarrolladas en diferentes espacios académicos, como congresos nacionales tanto para estudiantes como para profesionales además de las revistas contables que han tenido gran trayectoria como: Cuadernos de contabilidad, LUMINA, Revista Contaduría-Universidad de Antioquia, Revista internacional Legis, que han permitido hacer visibles las investigaciones para el desarrollo del saber contable, también se encuentran las mismas universidades y entidades como el Colegio de contadores Públicos de Colombia, Instituto Nacional de Contadores Públicos, la Junta Central de Contadores, que ha permitido espacios de discusión para el desarrollo la investigación en Contable. A continuación se presentan los resultados obtenidos del estudio cualitativo realizado, para evidenciar las tendencias de investigación que ha tendido la Contabilidad en el periodo de 2011 a 2015, teniendo en cuenta las variables que se han determinado:

\section{$4.1 \quad$ Idioma}

La ilustración 2 muestra el idioma en los que esta publicados los artículos de las revistas seleccionadas: Ilustración 2. Idioma

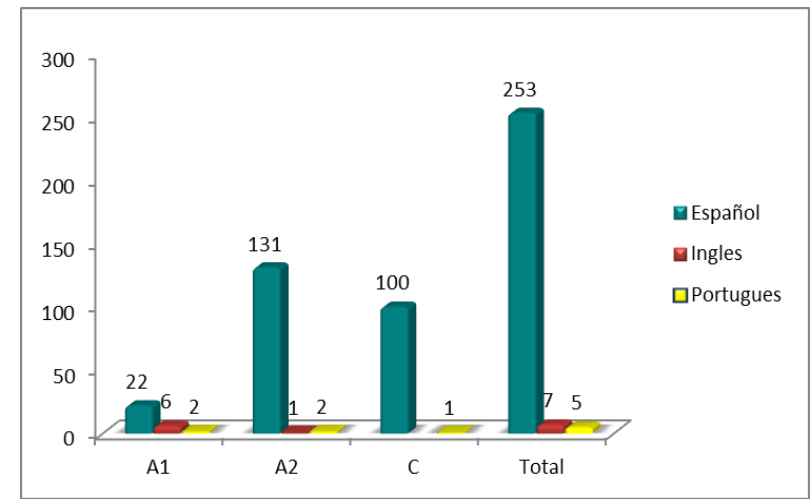

Fuente: elaboración propia 
En el grafico 2, se destaca que la mayoría de los artículos son publicados en español con un total de 253 de 265 artículos revisados. Se evidencia que el número de las revistas que publica en inglés son las revistas que se encuentran en la categoría A1, el total de las publicaciones en inglés hacienden a siete. En cuanto al idioma

Portugués, hay un total de cinco publicaciones distribuidas en cada una de las categorías de las revistas.

Dentro de la reflexión propuesta para esta categoría, se destaca la importancia de empezar a publicar documentos en inglés ya que esto permite tener mayor visibilidad en países extranjeros. Pacheco \& Hurtado (2013), destacan que las principales revistas indexadas a nivel internacional publican textos exclusivamente en inglés, pues facilitan a los motores de búsqueda identificar de manera fácil el tema desarrollado en la investigación.

En el estudio realizado por Castillo en 2011, titulado "El rol de las publicaciones científicas en la comunicación del EEES: indexación e impacto". En dicha investigación, Castillo (2011, citado por Pacheco \& Hurtado, 2013) describe a las revistas científicas como un instrumento de poder pues su edición se centra en los países desarrollados. Castillo basa su investigación en los resultados del Journal Citation Reports y en la notoria participación que tienen en este reporte las grandes casas editoriales, entre las que se destacan: Wiley-Blackwell Publishing, Sage Publications, Oxford University Press.

\subsection{Países}

La ilustración 3, evidencia que hay un $67.5 \%$ de las publicaciones colombianas con respecto a otros países que publican en estas revistas. Por su parte, España al ser un país como referente en investigación contable en Colombia, se ubica en un segundo lugar con el $12.1 \%$. Las publicaciones que se hacen en colaboración con las universidades extranjeras a pesar que de la muestra solo tiene un $2.6 \%$ indica que hay un gran interés en hacer visible la investigación realizada en Colombia a nivel internacional, dentro de los países en donde se encuentran estos convenios son España y México. COLCIENCIAS en 2016, dentro de las políticas adoptadas para mejorar la calidad de las publicaciones científicas nacionales, destaca el tema de "la interacción y colaboración internacional de los investigadores", todo esto a causa del incremento exponencial de las tecnologías de la información que ha facilitado acceso a nuevo conocimiento a nivel mundial.
Ilustración 3. Países

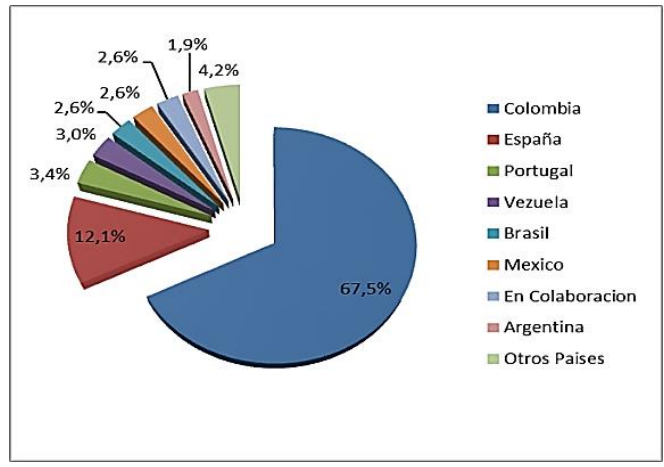

Fuente: elaboración propia

4.3 Ranking Universidades Colombianas

Tabla 2. Ranking Top 10 Universidades Colombianas

\begin{tabular}{|c|c|c|}
\hline N & Universidal & $\begin{array}{c}\text { Núm } \\
\text { Publicaciones } \\
\text { 2011-2015 }\end{array}$ \\
\hline & Pontificia Universidad Javeriana & 20 \\
\hline 2 & Universijad IVa: onal de & 15 \\
\hline 3 & Universijad de A.ntioquia & 11 \\
\hline 4 & Universiyad de Man zales & 10 \\
\hline 5 & Universidad de Medelin & 9 \\
\hline 6 & Universidad del $v_{\exists}$ le & 9 \\
\hline T & Universisad de la $\subseteq$ ale & 8 \\
\hline 8 & Unirersidad Libre de Colorbie & $i$ \\
\hline 9 & $\begin{array}{l}\text { Universidad Miltar Nueva } \\
\text { Grenada }\end{array}$ & $i$ \\
\hline 10 & $\begin{array}{l}\text { Polté: cc Jolombiano Jaime } \\
\text { Isaza Cadavid }\end{array}$ & 6 \\
\hline
\end{tabular}

Fuente: elaboración propia

Por otra parte, la tabla 2 presenta el Ranking de las universidades que publican sus investigaciones, la muestra comprende 130 Universidades, entre las cuales se encuentra 43 Universidades Colombianas, 69 extranjeras y 18 convenios de universidades Colombianas y extranjeras.

El primer puesto lo ocupa La Pontificia Universidad Javeriana, esto se debe a que es una de las Universidades a nivel nacional, dedicada exclusivamente a las publicaciones de la ciencia contable, además, su mayor interés es publicar sus investigaciones en este campo. La Universidad Nacional de Colombia, así como la Universidad de Antioquia, ocupan el segundo y tercer lugar respectivamente. Estás universidades son consideradas como grandes referentes contables a nivel nacional y se encuentran dentro de las primeras universidades que generan las investigaciones en contabilidad. La universidad Militar Nueva granada, como nuestra casa de estudio, ha logrado entrar en la dinámica de investigación en Contabilidad y se encuentra en el puesto siete. 


\subsection{Líneas de Investigación}

La ilustración 4 presenta "las líneas de investigación", en primer lugar y tercer lugar se destaca la línea de "investigación y educación contable" con un $20.75 \%$ así como la línea de "Teoría contable" con un $15.47 \%$, esto se debe al gran interés en el desarrollo del saber contable propuesto por González en 2005, quien además realiza una importante critica en cuanto a que;

"Aun hoy persiste la preocupación por los peligros que representa para el desarrollo del saber, que los contables sigan obnubilados frente al curso tomado por la ciencia y sin reflexión alguna los adopten en la contabilidad. El mejor antídoto contra la adaptación, copia y trasplante no es otro que la creatividad, el ingenio la reflexión y la implementación de los procesos de investigación en contabilidad" González (2005, p.5).

La segunda línea de gran interés es la de "Contabilidad internacional", esto se debe a la preocupación por la implementación de las NIIF en Colombia con la expedición de la Ley 1314 de 2009 en la cual se regulan los principios y normas de contabilidad e información financiera en Colombia y los Decretos 2784, 2706 y 3022 de 2013 los cuales adoptan oficialmente dichos estándares.

llustración 4. Líneas de Investigación

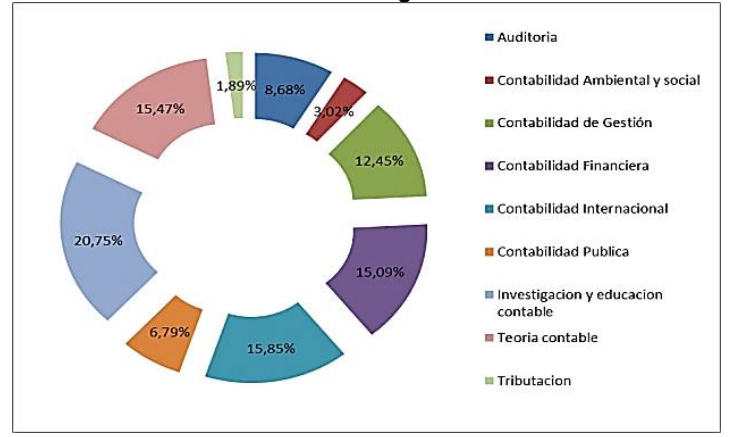

Fuente: elaboración propia

4.5 Metodologías de Investigación

llustración 5. Metodología de Investigación

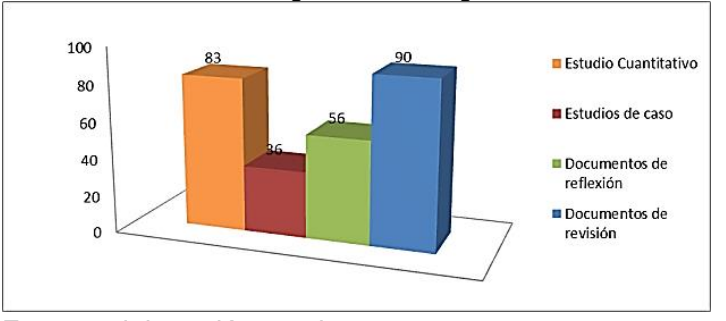

Fuente: elaboración propia
A Partir de la metodología utilizada en cada uno de los artículos, en los resultados obtenidos, se destaca que los investigadores usan más los estudios cuantitativos y los

documentos de reflexión, estos constituye una mayor compresión para el objeto de estudio.

Aunque la metodología de estudio de caso está en el último lugar de las cuatro categorías seleccionadas, para el desarrollo de la presente investigación, es importante destacar que los estudios casos están en la investigación cualitativa de tipo interpretativa, esto comprende esquemas para el desarrollo de las prácticas contables en contextos sociales y organizativos de los cuales forma parte. Ryan et.al (2004), consideran que los estudio de casos ofrecen la posibilidad de comprender la naturaleza de la contabilidad en la práctica, tanto teoría como en la técnicas, procedimientos y sistemas usados, así como en la forma en que se usan.

Dichos autores, destacan cinco tipos de estudio de casos en lo contable: estudio de casos descriptivos, ilustrativos, experimentales, exploratorios y estudio de casos explicativos, sin embargo para el estudio realizado, se tomó en cuenta el estudio de casos de tipo exploratorio, este tipo de caso permite explorar las razones de determinadas prácticas contables, se pueden generar hipótesis sobre los motivos de una práctica concreta y generalmente la pretensión es elaborar suposiciones que se pueden someter más adelante en una rigurosa comprobación empírica.

\subsection{Artículos publicados en revistas}

llustración 6. Porcentaje de artículos publicados en revistas

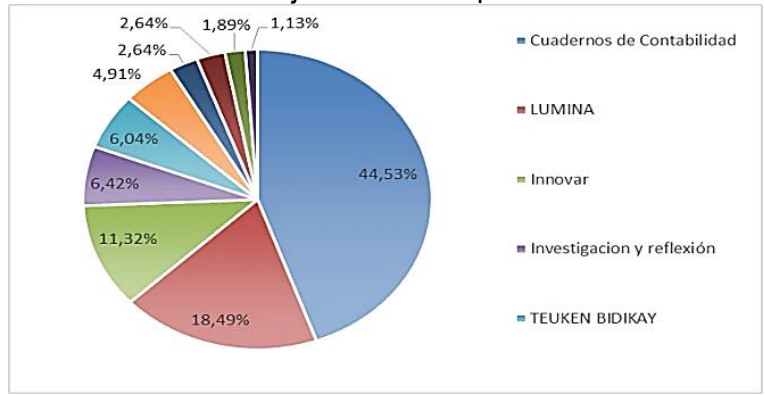

Fuente: elaboración propia

La revisa Cuadernos de Contabilidad como la revista LUMINA, al ser las dos revistas indexadas y categorizadas en COLCIENCIAS dedicadas exclusivamente a publicaciones contables, presentan más de la mitad de la muestra seleccionada, con un $44.53 \%$ y $18.49 \%$ respectivamente. Una estrategia muy válida para el 
fortalecimiento de las revisas de investigación exclusivas en publicaciones contables es la Red Colombiana de Editores y Revistas Contables (REDITORES), que como se

Mencionó con anterioridad, busca cooperación académica entre las universidades para el fortalecimiento de la investigación en contabilidad.

También es importante integrar entidades 0 agremiaciones como el Instituto de Contadores Públicos, el Colegio de Contadores, así mismo, es significativo contar con entidades como la Junta Central de Contadores Públicos como tribunal disciplinario y el Concejo Técnico de la Contaduría Pública como entidad encargada de la orientación técnica-científica de la profesión y de la investigación de los principios de contabilidad y normas de auditoría de aceptación general en el país. Se considera, que este tipo de entidades cuentan con muy poca participación en publicaciones científicas.

\subsection{Publicaciones Anuales 2011-2015}

Las publicaciones científicas en las ciencias contables han tenido un auge significativo puesto que ha habido una mayor preocupación por los profesionales contables en hacer visible sus investigaciones. La ilustración 7 muestra el número de publicaciones científicas anuales entre 2011 a 2015 y puede justificarse con la propuesta de Gómez (2003), el cual manifiesta que la contabilidad se encuentra en una etapa germinal de difusión y desarrollo disciplina.

Para el año 2013 se observa un mayor número de publicaciones, lo cual se puede sustentar en la preocupación latente ante la implementación de la normativa internacional (NIIF), además de una fuerte reflexión en torno a concientizar al contador de que no todo debe ser "generar valor al accionista" e involucrarse en procesos que generen sostenibilidad para las empresas, en otras palabras identificar la función social tanto de la contabilidad como del profesional contable.

Ilustración 7. Número de Publicaciones Anuales 20112015

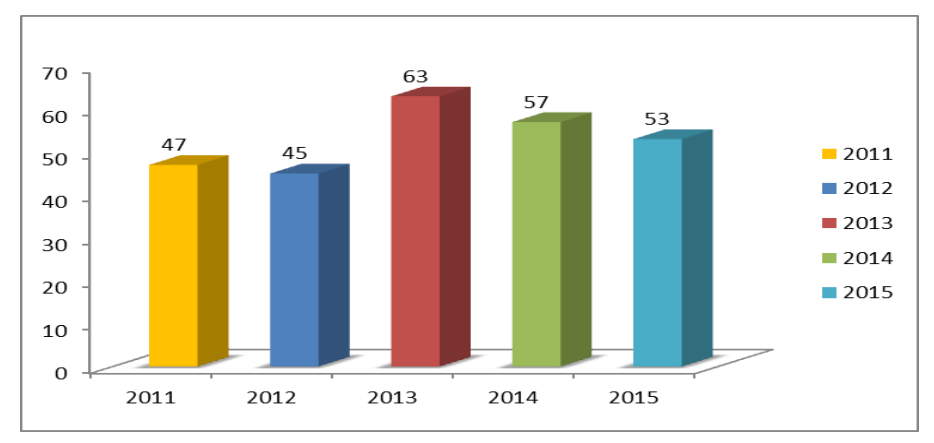

Fuente: Elaboración Propia

\section{CONCLUSIONES:}

Este estudio presenta el desarrollo histórico que ha tenido la investigación en contabilidad desde autores como Franco (1998), Cañibano \& Angulo (1996), (1999), Millan (1999), Gonzales (2005), Gómez, (2003), Machado (2012) , Aquel \& Cicerchia (2012), Salazar (2015), dentro del cual se puede facilitar el entendimiento a quien quiera comprender a cerca de las miradas ortodoxa y heterodoxa, los programas de investigación, un marcado enfoque del desarrollo de la contabilidad desde las ciencias sociales, además, una sencilla pero clara contextualización en cuanto a la relación de la teoría y práctica. Por último, los antecedentes se tomaron en cuenta para entender el desarrollo que ha tenido la investigación en contabilidad.

La base principal la investigación, fue revisar las publicaciones científicas, en las cuales se evidencia un crecimiento de las publicaciones en temas contables, además de la preocupación de las exigencias de COLCIENCIAS ante las políticas de publicación científicas como el idioma en que se publica y hacer los trabajos en colaboración con investigadores de otros países. Estas solicitudes no son algo que se deba cumplir por puntos, sino como algo que obliga a mejorar la calidad en los estándares y participación en la comunidad global. Por otra parte se realiza el desarrollo en torno al fortalecimiento de las revistas contables colombianas lo cual evidencia mayor visibilidad a nivel internacional y nacional que deja en claro que la investigación contable que se están realizando está proyectada al fortalecimiento del saber contable.

Dentro de los resultados encontrados se identifican las tendencias de investigación en Colombia; teoría o práctica, se demuestra un gran aporte de las investigaciones relacionados a los estudios de caso a la contabilidad y desde lo teórico como lo práctico. Las anteriores afirmaciones se ven reflejadas en el incremento de publicaciones con relación a las líneas de investigación en contabilidad financiera y contabilidad internacional.

Finalmente, con este trabajo de recopilación y análisis de tendencias en publicaciones desde revistas dedicadas a la ciencia contable se pretende evidenciar y dar una herramienta para que no solo los académicos contables realicen publicaciones sino también invitar y mostrar los canales de publicación para que los profesionales contables se animen a hacer parte del fortalecimiento y reflexión en su carrera, la importancia es la necesidad de hacer investigaciones en contabilidad ya sea en estudios de reflexión, estudios empíricos o estudios de caso los cuales seguramente darán como resultados el aporte de diferentes puntos de vistas en pro de facilitar múltiples 
alternativas a problemáticas actuales, las que además permitirán un aporte adicional al saber contable tanto en lo teórico como también en lo práctico, con estos procesos se conseguirá generar una investigación con mayor potencial.

\section{REFERENCIAS:}

Anes, R. D. (1996). Una contribución a la historia de la contabilidad: análisis de las prácticas contables desarrolladas por la tesorería de la Casa de la Contratación de las Indias de Sevilla (15031717) (Vol. 32). Universidad de Sevilla.

Aquel, S., \& Cicerchia, L. (2012) Perspectivas en investigación contable: el enfoque crítico interpretativo. Decimoséptimas Jornadas "Investigaciones en la Facultad" de Ciencias Económicas y Estadística. Universidad Nacional de Rosario, Argentina. Noviembre de 2012.

Araya (2002). Las representaciones sociales: ejes teóricos para su discusión. : Cuaderno de Ciencias sociales 127. San José, Costa Rica: Facultad Latinoamericana de Ciencias sociales.

Bardales, C. T., \& Wong, A. L. (2012). Los fundamentos epistemológicos de la contabilidad y su incidencia en la formación competitiva del contador público.Sotavento MBA, (19), 32-50.

Cañibano, L., \& Angulo, J. G. (1996). Los programas de investigación en contabilidad. Contaduría Universidad de Antioquia, (29), 13-62.

Córdoba, J (2014). Aproximación al desarrollo científico de la contabilidad: un recorrido por el pensamiento contable. Revista Unimar, 28(4).

Franco, R. (1995). Contabilidad integral, teoría y normalización. Pereira, Colombia: Investigar.

Larrinaga, C. (1999). Alternative Perspectives in Accounting Research: A Review (Perspectivas alternativas de investigación en contabilidad: Una revisión). Alternative Perspectives in Accounting Research: A Review (Perspectivas alternativas de investigación en contabilidad: Una revisión), 103-131.

León, H. A. R. (2014). Problemas de investigación en contabilidad y problemas de la investigación en contabilidad. CLIO AMERICA, 8(15), 75-82.

López, S. M. M., Rojas, G. A. R., \& Ramírez, H. J. S. (2014). Didácticas para la formación en investigación contable: una discusión crítica de las prácticas de enseñanza. Revista Facultad de Ciencias Económicas, 23(1), 53-86.

Luna, E. G., Navas, D. F., Mayor, G. A., \& Buitrago, L. A. B. (2014). Metodología para la revisión bibliográfica y la gestión de información de temas científicos, a través de su estructuración y sistematización. DYNA: revista de la Facultad de Minas. Universidad Nacional de Colombia. Sede Medellín,81(184), 158-163.

Machado Rivera, M. A., Castaño Rios, C. E., Guevara Sanabria, J. A., Osorio Agudelo, J. A., Zamarra Londoño, J., Muñoz Osorio, L. M., ... \& Saavedra García, M. L. (2016). Caminos contables: problemas y metodologías para el desarrollo de la investigación.

Macías, H. A. (2013). Internacionalización de las revistas contables: un aporte al debate polifónico. Cuadernos de Contabilidad, 14(35), 785-794.

Macias, H. A. (2016). La investigación contable colombiana frente a los nuevos criterios de medición de Colciencias. EN-CONTEXTO, 4(4), 39-48.

Macías, H. A., Gómez, M., \& León, E. (2012). Las revistas académicas como plataforma para la construcción de conocimiento contable. Revista Facultad de Ciencias Económicas: Investigación y Reflexión, 20(2), 5-9.

Macías, Hugo A. (2013). Debate sobre el presente y el futuro de las revistas contables colombianas: acuerdos, desacuerdos y retos. Cuadernos de Contabilidad, 14 (34), 265-271.

Macías, Hugo A. (2013). Vínculos de la investigación contable interpretativa con la producción académica colombiana: avances y oportunidades. Cuadernos de Contabilidad, 14 (35), 699-727.

Martínez Carazo, P. C. (2011). El método de estudio de caso Estrategia metodológica de la investigación científica. Revista científica Pensamiento y Gestión, (20).

Naranjo, O. (2015). La investigación contable: antecedentes, contexto epistemológico y tendencias axiológicas para restituir la confianza social.Unaciencia, (6).

Ortega, N. K. F. (2013). La contabilidad vista como dispositivo de poder: aproximación 
interpretativa desde la perspectiva foucaultiana. Cuadernos de Contabilidad, 14(34).

Quijano-Valencia, O. (2002). Nuevos tiempos, nuevas competencias. De las mono-competencias a la policognición. Del hacer al saber. Realidades y perspectivas de la educación cantable en Colombia. Popayán: Universidad del Cauca

Restrepo, C. M. V. (2013). Caracterización de la investigación contable. El aporte de Chua. Revista Lúmina, (14).

Rios, C. E. C. (2016). El nacimiento de la Red Colombiana de Editores y Revistas Contables. Contaduría Universidad de Antioquia, (67), 11-15.

Rivera, M. A. M. (2013). Del positivismo a la diversidad epistemológica: un paso necesario para la construcción de pensamiento contable. Contaduría Universidad de Antioquia, (60), 219237.

Ryan, B., Scapens, R. W., \& Theobald, M. (2004). Metodología de la investigación en finanzas y contabilidad. Deusto.

Sampieri, R. H., Collado, C. F., \& Lucio, P. B. (1996). Metodologia de la investigaci6n. Edici6n McGraw-Hill.

Santos, L. A. (2005). Fundamentación conceptual de la crisis contable. Revista Internacional de Legis Contabilidad y Auditoría, 21, 151-192.

Tarazona, A. H., \& Martínez, J. A. P. (2013). Estrategias para mejorar el impacto de las publicaciones indexadas en Publindex, ISI, Scopus y SciELO: el caso de la Universidad Santo Tomás, Colombia. Hallazgos, 10(19). Valero-Zapata, G. M., \& Patiño-Jacinto, R. A. (2012). Los grupos de investigación contable reconocidos por Colciencias. Cuadernos de Contabilidad, 13(32), 175-201.

Villegas, M. G. (2003). Algunos comentarios sobre la potencialidad de la investigación en contabilidad. Innovar: Revista de ciencias administrativas y sociales, 139-144. 